\title{
Adaptación y validación argentina de una versión breve de la Escala de Parentalidad Positiva (E2p)
}

\section{Argentine Adaptation and Validation of a Short Version of the Positive Parenting Scale (E2p)}

\author{
Jael Vargas-Rubilar, b,*, Viviana Noemí Lemos ${ }^{\mathrm{a}, \mathrm{b}}$, María Cristina Richauda, b, \\ ${ }^{a}$ Consejo Nacional de Investigaciones Científicas y Técnicas, Argentina \\ ${ }^{\mathrm{b}}$ Centro Interdisciplinario de Investigaciones en Ciencias de la Salud y del Comportamiento, \\ Universidad Adventista del Plata, Argentina
}

Recibido: 24 de julio de 2020

Aceptado: 30 de noviembre de 2020

\section{Resumen}

Antecedentes: la parentalidad positiva se caracteriza por promover relaciones saludables en la familia basadas en el ejercicio de la responsabilidad parental, garantizar los derechos de los hijos y optimizar su potencial desarrollo y bienestar. Dada la importancia de esta temática para el buen desarrollo de los niños, es fundamental contar con instrumentos adecuados para su evaluación. Objetivo: adaptar y validar a la Argentina una versión breve de la Escala de Parentalidad Positiva (E2p) de Gómez y Muñoz (2014). Método: la escala fue administrada a una muestra de 546 padres y madres (edad: $M=38.54 ; D E=6.37$ ). Se estudió el funcionamiento de los ítems, se realizaron análisis factoriales confirmatorios para establecer si la estructura factorial de la forma breve permanecía estable con respecto a la de la forma completa y se calculó la consistencia interna de la escala. Resultados: el modelo factorial propuesto fue puesto a prueba a través de un AFC con el método ULS que indicó un ajuste muy satisfactorio del modelo abreviado. La consistencia interna de la prueba fue adecuada para la escala total y por dimensiones. Conclusiones: Finalmente, se discuten los hallazgos, limitaciones y futuras líneas de investigación.

Palabras clave: parentalidad positiva; evaluación; escala abreviada; validación psicométrica.

Para citar este artículo:

Vargas-Rubilar, J., Lemos, V. N., \& Richaud, M. C. (2020). de Parentalidad Positiva (E2p). Liberabit, 26(2), e413. https:// doi.org/10.24265/liberabit.2020.v26n2.08 Adaptación y validación argentina de una versión breve de la Escala

\begin{abstract}
Background: Positive parenting is characterized by promoting healthy relationships in the family based on parental responsibility to ensure children's rights and optimize their potential development and well-being. Given the importance of this topic for the good development of children, it is essential to have adequate instruments for its evaluation. Objective: to adapt to and validate in the Argentine population a short version of the Positive Parenting Scale (E2p) by Gómez and Muñoz (2014). Method: the scale was administered to a sample of 546 fathers and mothers (age: $M=38.54 ; S D=6.37$ ). The functioning of the items was studied, confirmatory factor analyses were carried out to establish whether the factor structure of the short form remained stable with respect to the complete form, and the internal consistency of the test was analized. Results: the proposed factorial model was tested through a confirmatory factor analysis (CFA) with the unweighted least squares (ULS) method, which indicated a very satisfactory fit of the abbreviated model. The internal consistency of the test was adequate for the full scale and dimensions. Conclusions: finally, the findings, limitations and future lines of research are discussed.
\end{abstract}

Keywords: positive parenting; evaluation; abbreviated scale; psychometric validation.

Este es un artículo Open Access publicado bajo la licencia Creative Commons Atribución 4.0 Internacional. (CC-BY 4.0)

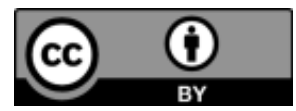

Universidad de San Martín de Porres, Lima - Perú * psicojael@gmail.com 


\section{Introducción}

El interés por el estudio de la parentalidad o función de crianza, se ha incrementado en las últimas décadas, convirtiéndose en objeto de diversas investigaciones científicas. No obstante, dicho estudio tiene sus orígenes en la teoría del apego y el enfoque de los estilos parentales (Vargas-Rubilar \& Richaud, 2018).

Según Bowlby (1969) -autor de la teoría del apego-, la conducta de crianza no es el resultado de un instinto invariable, así como tampoco resulta razonable considerarla solo como un producto del aprendizaje. Desde su perspectiva, la crianza tiene fuertes raíces biológicas, lo que explica las fuertes emociones asociadas a ella. Sin embargo, el estilo que adopta depende en gran parte de las experiencias vividas durante la infancia y la adolescencia, tanto por los padres como por cada hijo en forma individual. Por lo tanto, la capacidad de las figuras parentales para vincularse con sus hijos e hijas depende tanto de su potencial biológico como de las experiencias infantiles de apego con sus propios padres u otros adultos significativos.

Los estudios posteriores de Baumrind (1978) mostraron que la crianza se transmite a través de la socialización, que es un proceso iniciado por los adultos y a través del cual los niños y las niñas aprenden creencias, valores socioculturales y hábitos, por medio de la educación, el entrenamiento y el aprendizaje social o modelado. Consecuentemente, los padres o cuidadores pueden tener una influencia relevante sobre la personalidad, el carácter y las competencias de sus hijos (Bornstein \& Bornstein, 2007; Dwairy \& Dor, 2009).

Años más tarde, para denominar la función de crianza, se acuña el término parentalidad (parenting en inglés) que, como el vocablo lo indica, se refiere al parentesco o parentela, es decir, a los lazos de alianza o consanguineidad entre padres e hijos e hijas al interior de las familias (Vargas-Rubilar \& Richaud, 2018). Sin embargo, la parentalidad se distingue del parentesco en tanto que puede incluir o no lazos sanguíneos, como en el caso de adopción o crianza de hijos no biológicos (Solís-Ponton et al., 2006).

Desde esta perspectiva, la crianza de los hijos implicaría un proceso biológico y social complejo, que va mucho más allá de brindar la provisión de alimentos y seguridad a los descendientes. La parentalidad se refiere a las actitudes y acciones llevadas a cabo tanto por la madre como por el padre en el proceso de socializar, cuidar, educar y preservar a sus hijos e hijas (Barudy \& Dantagnan, 2010).

Desde sus inicios, la mayor parte del estudio de la parentalidad se abocó al análisis de la primera infancia, seguido por el de las etapas de la niñez y adolescencia (Bornstein \& Bornstein, 2007). Al respecto, la investigación psicológica ha documentado un importante número de trabajos teóricos y empíricos que destacan el importante rol de la socialización parental en el ajuste psicosocial en la infancia, en la niñez (Vargas-Rubilar \& Richaud, 2018) y en la adolescencia (Dwairy \& Dor, 2009).

Particularmente, la parentalidad positiva hace referencia al comportamiento de los padres basado en el interés superior del niño o la niña. Según esta conceptualización, una parentalidad apropiada se caracteriza por «promover relaciones positivas en la familia, fundadas en el ejercicio de la responsabilidad parental para garantizar los derechos de los hijos y las hijas, y optimizar su desarrollo potencial y su bienestar» (Martín et al., 2009, p. 22). De esta manera, según lo mencionado por algunos estudios (Durrant, 2011; Kyriazos \& Stalikas, 2018; Sanders, 2008; Sanders \& Mazzucchelli, 2018; Pickering \& Sanders, 2016), el ejercicio de una parentalidad positiva requiere la conjunción equilibrada de diversos componentes imprescindibles, tales como: el apego seguro, la empatía, la comunicación, la expresión del afecto, la disciplina positiva y la autonomía otorgada a los hijos e hijas, en las distintas etapas del ciclo vital. Este cambio de enfoque en relación a la crianza fue seguido por la Declaración de los Derechos del Niño 
de las Naciones Unidas (United Nations Foundation [UNF], 2006).

Los estudios en el área han analizado la parentalidad positiva desde el enfoque de las competencias parentales, dado que el mismo permite estudiar y promover los recursos con los que cuentan los padres o cuidadores para ejercer la función de crianza (e.g, Gómez \& Muñoz, 2014; Rodrigo et al., 2010, Rodrigo et al., 2015, Torío-López et al., 2019). En este sentido, Bronfenbrenner y Evans (2000) han definido la competencia parental como la «adquisición demostrada, y posterior desarrollo, de conocimientos y capacidades para conducir el comportamiento parental propio a través de diversas situaciones de la crianza en las distintas dimensiones (i.e., física, cognitiva, socioemocional, entre otras) del desarrollo de los hijos e hijas» (Bronfenbrenner \& Evans 2000, citado en Gómez \& Muñoz, 2014, p. 6).

Asimismo, autores como Barudy y Dantagnan (2010), aluden a las competencias parentales como «las capacidades prácticas que tienen las madres y padres para cuidar, proteger, educar a sus hijos y asegurarles un desarrollo suficientemente sano» (p. 34). Así, la posibilidad de contar con estas competencias estaría influida por componentes biológicos-hereditarios, modulados por experiencias vitales, la cultura y los contextos sociales en los que se ha desenvuelto el progenitor, tanto en el presente como en el pasado (Belsky, 2015; Bornstein, 2007; Bronfenbrenner \& Evans, 2000).

Los principales ámbitos en los que se han desarrollado trabajos sobre competencias parentales son los siguientes: (a) judicial, como medio para determinar la separación o custodia de los niños y las niñas de los padres, madres o cuidadores que los tienen a cargo (e.g., Cirillo, 2013) y (b) en contextos de riesgo psicosocial, ya sea para promover programas de prevención o asistencia para familias socialmente vulnerables (e.g., Esteban \& Firbank, 2019; Rodrigo et al., 2015; Torío-López et al., 2019; Vargas-Rubilar et al., 2017).

\section{Evaluación de las competencias parentales}

Aunque en los últimos años el estudio de las capacidades para la crianza ha tomado un visible auge en las publicaciones científicas, aún son insuficientes los trabajos acerca de su estudio y medición en población latinoamericana.

Por ejemplo, Martín et al. (2013) construyeron la Escala de Competencia y Resiliencia Parental para contextos de riesgo social con buen funcionamiento en población española. Dicho instrumento valora diez habilidades parentales vinculadas al desarrollo y autonomía personal, la organización doméstica, la búsqueda de apoyo, las competencias cognitivas y emocionales, entre otras. Asimismo, Suárez et al. (2016), validaron la Escala de Parentalidad Positiva (EPP) para evaluar programas presenciales y online de apoyo parental en población española que mide las áreas de implicación familiar, afecto y reconocimiento, comunicación y control de estrés y actividades compartidas.

Por su parte, Martínez-González e Iglesias-García (2018) realizaron la validación de la Escala de Competencias Parentales para padres españoles con niños(as) pequeños(as) (i.e., Parenting Competence scale for parents with young children [PCS-Y]). Este instrumento evalúa las siguientes dimensiones: autorregulación, autoestima, promoción de la autoestima de los niños, imposición para la resolución de conflictos y comunicación no asertiva. También, Iglesias-Garcia et al., (2019) llevaron a cabo la validación de la Escala de Competencias Parentales Emocionales con Hijos(as) Adolescentes (ECPE-HA) que valora las siguientes áreas: control y relajación, asertividad en el rol parental, imposición y promoción de la autoestima del adolescente.

Anteriormente, Bayot y Hernández (2008) propusieron la Escala de Valoración de las Competencias Parentales Percibidas (ECPP). Estos autores definieron las competencias parentales percibidas como la capacidad autopercibida de los padres y madres para enfrentar la crianza de sus hijos 
e hijas adecuadamente. La ECPP evalúa cinco factores o dimensiones, estos son los siguientes: implicación escolar, dedicación personal, ocio compartido, asesoramiento/orientación, asunción del rol de ser padres. Los ítems son proposiciones relacionadas con la función de ser padre/madre/pareja. Debido a que la ECPP cuenta con la posibilidad de valorar ambas perspectivas (i.e., padres e hijos(as). Azar et al. (2019) llevaron a cabo la validación de esta escala en Argentina, evidenciando un adecuado funcionamiento psicométrico para cuatro factores. No obstante, este instrumento no incluye la valoración de algunas habilidades fundamentales para la crianza, como el apego, la sensibilidad parental, la disciplina positiva, ni el autocuidado parental.

Por otra parte, Gómez y Muñoz (2014) desarrollaron en Chile la Escala de Parentalidad Positiva (E2p), cuyo objetivo es identificar aquellas competencias parentales que los padres, madres o cuidadores utilizan al relacionarse con sus hijos e hijas. La E2p se basa en cuatro enfoques teóricos interconectados: el de parentalidad positiva (Rodrigo et al., 2015), la teoría ecosistémica del desarrollo humano (Bronfenbrenner, 1987), la teoría del apego (Bowlby, 1969) y la teoría de la resiliencia familiar (Walsh, 2004). De acuerdo a este enfoque teórico, las competencias parentales son el conjunto de conocimientos, actitudes y prácticas de crianza vinculares, formativas, protectoras y reflexivas; aprendidas y actualizadas a partir de una historia y las oportunidades que ofrece la ecología de la parentalidad. Desde este enfoque, las competencias parentales favorecen la organización de la propia experiencia y el comportamiento parental a través de diversas situaciones habituales de la familia y la crianza de los hijos, conduciendo, preservando y promoviendo trayectorias de desarrollo positivo en los hijos e hijas, con el propósito de garantizar su bienestar y el pleno ejercicio de sus derechos (Gómez \& Contreras, 2019). La versión original de la E2p (Gómez \& Muñoz, 2014) se compone de 54 reactivos que dan cuenta de comportamientos cotidianos de crianza que estarían reflejando el despliegue de la competencia parental en cuatro áreas:
1. Competencias vinculares, es decir, aquellas orientadas a promover un vínculo de apego seguro y un apropiado desarrollo socioemocional en los niños y las niñas. Los principales componentes de esta área son los siguientes: la mentalización, la sensibilidad parental, la calidez emocional, el involucramiento parental en los distintos aspectos de la vida cotidiana del niño o la niña.

2. Competencias formativas, es decir, las que contribuyen al desarrollo del aprendizaje y socialización de los niños y las niñas. Sus componentes son los siguientes: la orientación la estimulación del aprendizaje, guía y consejo en diversas situaciones del ciclo vital, el establecimiento de reglas y rutinas mediante una disciplina positiva basada en los buenos tratos a los hijos e hijas.

3. Competencias protectoras, son aquellas orientadas a cuidar y resguardar debidamente a los hijos(as), satisfaciendo sus necesidades básicas, garantizando sus derechos y favoreciendo su integridad física, emocional, social y sexual. Sus principales componentes son los siguientes: provisión de cuidados para la satisfacción de las necesidades primarias, garantías de seguridad física, emocional y psicosexual y la organización de la vida cotidiana de los niños y niñas.

4. Competencias reflexivas, son las que permiten recapacitar sobre las influencias y trayectorias de la propia parentalidad, monitorear las prácticas de crianza actuales y evaluar el desarrollo del hijo/a. Está conformada por componentes como prever situaciones de la crianza o posibles contextos adversos; así como monitorear las influencias biopsicosociales sobre el desarrollo del niño o la niña, el autocuidado parental, la meta-parentalidad o habilidad para reflexionar en tres áreas interrelacionadas: la historia de parentalidad vivida, las prácticas parentales actuales y la calidad de la relación parento-filial. 
Consideramos que el enfoque teórico operacionalizado en la E2p es novedoso y explicativo del constructo a evaluar, dado que incluye aspectos vinculares o afectivos, de disciplina positiva, de cuidado o protección y de la autopercepción del rol parental que han sido identificados como esenciales por diversos estudios sobre parentalidad positiva (Rodrigo et al., 2015; Sanders, 2008; Sanders \& Mazzucchelli, 2018).

Una de las limitaciones para el estudio de la parentalidad percibida en el contexto argentino ha sido el insuficiente número de instrumentos psicométricos construidos o validados para analizar los distintos aspectos de este constructo tanto desde la mirada de los hijos como de la de los padres. En Argentina, los instrumentos que operacionalizan la parentalidad percibida se han focalizado principalmente en la valoración de los estilos parentales y el apego (VargasRubilar \& Richaud, 2018). Actualmente, siguen siendo escasas las medidas en español que permitan valorar las competencias parentales percibidas desde un enfoque positivo.

No obstante, resulta imprescindible estudiar los instrumentos antes de su aplicación al contexto en que se van a utilizar, en este caso en la población argentina, entendiendo que un constructo psicológico no necesariamente se expresa de igual forma en las diferentes culturas y países (Fernández et al., 2011). Por otra parte, estas medidas, además de tener una gran utilidad para el área clínica, se utilizan con fines de investigación y suelen ser aplicadas junto a otros instrumentos. Por esta razón, la tendencia actual es evaluar constructos a través de versiones cortas con el propósito de facilitar la administración y evitar sesgos en las respuestas asociadas al cansancio de los participantes (Cupani et al, 2019).

Con base en lo expuesto, este estudio tiene como objetivo proponer una versión breve de la escala de la E2p de Gómez y Muñoz (2014), estudiando sus propiedades psicométricas en padres y madres argentinos de niños y niñas de 9 a 12 años de edad.
Este rango de edad fue elegido ya que este trabajo es parte de un proyecto de investigación más amplio que estudia variables del desarrollo en las etapas de niñez media y tardía.

\section{Método}

Se realizó un estudio instrumental, ya que el mismo estuvo abocado a la adaptación y análisis de funcionamiento psicométrico de una prueba psicológica (Montero \& León, 2007).

\section{Participantes}

Se evaluó una muestra constituida por 546 padres de niños(as) escolarizados(as) (74.8\% mujeres; edad: $M=38.54 ; D E=6.37)$. En cuanto a la escolaridad de los participantes, el 14\% informó estudios secundarios incompletos, el $27 \%$ estudios secundarios completos, el 54\% tenía estudios terciarios o universitarios y el resto, estudios de posgrado. Los participantes eran progenitores o cuidadores de niños y niñas de 9 a 12 años de edad $(M=10.59 ; D E=$ 1.21), residentes en las provincias argentinas de Buenos Aires (46.4\%), Mendoza (11.3\%), Santa Fe (31\%) y Entre Ríos (11.2\%).

Los participantes fueron seleccionados a través de un muestreo no probabilístico, por accesibilidad o conveniencia (Otzen \& Manterola, 2017).

\section{Instrumentos}

Junto con la escala en estudio, se administró una encuesta sociodemográfica, a fin de obtener datos relativos a edad, sexo, lugar de residencia y escolaridad de los(as) participantes.

La versión original de la Escala de Parentalidad Positiva E2p elaborada por Gómez y Muñoz (2014), tal como se ha mencionado anteriormente, valora cuatro áreas de competencia parental: vinculares, formativas, protectoras y reflexivas. Este instrumento cuenta con ocho versiones para padres y madres o cuidadores según la edad de los hijos (i.e., 0 a 3 
meses, 4 a 10 meses, 19 a 36 meses, 3 a 5 años, 6 a 7 años, 8 a 12 años y 13 a 17 años de edad) con cinco opciones de respuesta (desde nunca a siempre) en todas las versiones. Un estudio reciente de la versión para progenitores de niños y niñas de 8 a 12 años realizado por los autores (Gómez \& Contreras, 2019), informó un alfa de Cronbach de .93 para la escala total, y valores entre .73 y .87 para las diferentes dimensiones. El AFC realizado para dicha versión mostró un ajuste satisfactorio al modelo de 4 factores (i.e., $N=393$; X2 = $2059<.001$; CFI = .98; TLI = .98; RMSEA = .38). En Argentina, la versión original fue adaptada lingüísticamente y analizada preliminarmente respecto a su estructura factorial en padres argentinos de niños de 3 a 5 años (Molina \& Vargas-Rubilar, 2016), mostrando buenos indicadores de funcionamiento psicométrico.

Hasta el momento no se ha publicado ninguna versión abreviada de la E2p, tampoco existen trabajos respecto a su funcionamiento psicométrico en la población argentina, tarea a la que está abocada este estudio.

\section{Procedimientos}

\section{Procedimientos seguidos en la recolección de datos}

En todos los casos, los participantes fueron contactados a través del centro educativo al que concurrían sus hijos. Antes de la evaluación, se comunicaron los objetivos de la investigación junto con los recaudos éticos de confidencialidad y anonimato de los datos de los participantes (American Psychological Association [APA], 2010). Enseguida, se solicitó el consentimiento informado a los padres y madres. Cabe destacar que la administración de la escala se llevó a cabo en el marco de un proyecto de investigación que incluía tanto padres y madres como hijos(as). Razón por la cual, se solicitó a uno de los padres (o tutores) que completara la escala, solamente en función de su hijo o hija que participaba del estudio. La administración de los instrumentos fue individual y estuvo a cargo de psicólogos entrenados previamente.

\section{Procedimientos seguidos para la adaptación y estudio de la escala}

Para la adaptación y estudio de la versión argentina de la E2p se utilizó la siguiente secuencia metodológica:

1. Adaptación lingüística, en la que se revisó cada reactivo para ajustarlo al lenguaje de la población argentina.

2. Revisión por parte de 6 jueces expertos.

3. Aplicación a una muestra piloto de 30 padres y madres.

4. Ajustes en función de las sugerencias aportadas por los jueces y los padres.

5. Aplicación a una muestra de tipificación.

6. Análisis descriptivos de los ítems (media, desvío, asimetría y curtosis).

7. Análisis del poder discriminativo de los ítems a partir del índice de homogeneidad corregido (IHc).

8. Realización de tres Análisis Factoriales Confirmatorios (AFC) desde una matriz de correlaciones policóricas, método de extracción ULS con estimación robusta, recomendado para este tipo de matriz (Ferrando \& LorenzoSeva, 2014). Se realizaron a través del software LISREL 8.8 (Jöreskog \& Sörborn, 1993) con el objetivo de evaluar y comparar el ajuste a los datos del modelo de la versión completa (54 ítems) y dos versiones abreviadas (24 ítems), una de primer orden y una de segundo orden.

9. Análisis de la consistencia interna de la escala a través del coeficiente Omega (McDonald, 1999; Ventura-León \& Caycho-Rodríguez, 2017). 


\section{Resultados}

\section{Revisión de los jueces expertos y de la muestra piloto}

Para llegar a versión argentina definitiva de E2p se desarrolló la siguiente secuencia:

1) Seis jueces expertos evaluaron la claridad y nivel de comprensión de los ítems para el contexto argentino.

2) Se realizó una evaluación piloto con un grupo de 30 padres y madres, quienes contribuyeron con sus comentarios y sugerencias a optimizar la claridad y comprensión de los reactivos.

A partir de sus recomendaciones, se revisaron y modificaron los términos que eran poco familiares para los participantes.

Las sugerencias de los jueces expertos y de los padres habilitaron algunas modificaciones menores en 12 de los 54 reactivos originales de la escala. Por ejemplo, en la versión original se menciona Cuando mi hijo/a está irritable, puedo identificar las causas (ej.: me doy cuenta si está de mal genio porque está enfermo), este se cambió por Cuando mi hijo/a está irritable, puedo identificar las causas (ej.: me doy cuenta si está de mal humor porque está enfermo).

\section{Análisis de ítems: descriptivos, discriminación y homogeneidad}

En esta instancia, se hicieron análisis descriptivos para cada ítem (ver Tabla 1). El poder discriminativo a nivel de ítem, fue calculado con el índice de homogeneidad corregido. Como puede observarse en la Tabla 1, la mayoría de los reactivos (excepto cuatro) presentaron valores aceptables (mayores a .30, Kline, 2016).
Por otra parte, algunos de los reactivos alcanzaron valores de asimetría y curtosis mayores a \pm 2 , considerados inadecuados para realizar el cálculo de estadísticos paramétricos (Bandalos \& Finney, 2010; Muthén \& Kaplan, 1985) (Ver Tabla 1).

\section{Análisis factorial confirmatorio}

Dada la distribución no normal de algunas variables, el AFC fue realizado desde una matriz de correlaciones policóricas, utilizando el método de extracción de ULS con estimación robusta, recomendado para este tipo de matriz (Ferrando \& Lorenzo-Seva, 2014).

Para analizar la estructura factorial de la E2p, se llevaron a cabo tres AFC, donde los modelos puestos a prueba respondían a lo siguiente: (a) la versión completa de la E2p adaptada al contexto argentino (54 ítems), (b) la versión abreviada de la misma (24 ítems) y (c) un modelo de segundo orden de versión abreviada (24 ítems). Para establecer estos tres modelos, se realizó primero un AFC de la versión completa de la escala de acuerdo al modelo propuesto por los autores (Gómez \& Muñoz, 2014). Luego, se seleccionaron solo 6 ítems por factor según los reactivos que saturaban más alto en cada dimensión y que representaban más adecuadamente los diferentes aspectos del constructo, siguiendo de este modo criterios tanto empíricos como teóricos. Con este procedimiento se eliminaron 30 de los 54 ítems originales quedando un total de 24 reactivos (señalados en negrita en la Figura 1) que constituyeron la versión breve de la prueba.

$\mathrm{Al}$ realizar el AFC, tanto de la escala completa (ver Figura 1) como de la abreviada, se observó que las covarianzas entre los factores eran bastante elevadas, pudiendo indicar la existencia de una variable subyacente que unificara dichos factores. Por ello, se decidió poner a prueba un tercer modelo que incluyera un factor general de segundo orden (ver Figura 2). 
Tabla 1

Estadísticos descriptivos para el conjunto de 54 ítems

\begin{tabular}{|c|c|c|c|c|c|c|c|}
\hline & \multirow{2}{*}{$M$} & \multirow{2}{*}{$D E$} & \multicolumn{2}{|c|}{ Asimetría } & \multicolumn{2}{|c|}{ Curtosis } & \multirow{2}{*}{ IHc } \\
\hline & & & Estadístico & Error estándar & Estadístico & Error estándar & \\
\hline Ítem 1 & 2.66 & .812 & -.162 & .105 & -.569 & .209 & .459 \\
\hline Ítem 2 & 2.41 & .958 & -.075 & .105 & -.193 & .209 & .378 \\
\hline Ítem 3 & 2.53 & .993 & -.495 & .105 & -.321 & .209 & .357 \\
\hline Ítem 4 & 3.28 & .819 & -1.119 & .105 & 1.358 & .209 & .352 \\
\hline Ítem 5 & 2.99 & 1.010 & -.775 & .105 & -.082 & .209 & .419 \\
\hline Ítem 6 & 2.46 & 1.080 & -.339 & .105 & -406 & .209 & .362 \\
\hline Ítem 7 & 3.43 & .747 & -1.234 & .105 & -.094 & .209 & .449 \\
\hline Ítem 8 & 2.95 & .883 & -.511 & .105 & -.094 & .209 & .427 \\
\hline Ítem 9 & 3.31 & .947 & -1.456 & .105 & 1.760 & .209 & .367 \\
\hline Ítem 10 & 2.66 & .802 & -.228 & .105 & -.303 & .209 & .393 \\
\hline Ítem 11 & 3.49 & .710 & -1.232 & .105 & 1.059 & .209 & .460 \\
\hline Ítem 12 & 2.94 & .802 & -.228 & .105 & .353 & .209 & .376 \\
\hline Ítem 13 & 2.52 & .958 & -.208 & .105 & -.342 & .209 & .367 \\
\hline Ítem 14 & 3.74 & .612 & -2.682 & .105 & 7.549 & .209 & .371 \\
\hline Ítem 15 & 3.31 & .845 & -1.095 & .105 & .733 & .209 & .447 \\
\hline Ítem 16 & 3.68 & .578 & -1.788 & .105 & 2.694 & .209 & .480 \\
\hline Ítem 17 & 3.23 & .832 & -.895 & .105 & .436 & .209 & .532 \\
\hline Ítem 18 & 3.51 & .786 & -1847 & .105 & 3.740 & .209 & .494 \\
\hline Ítem 19 & 3.54 & .771 & -1.668 & .105 & 2.336 & .209 & .444 \\
\hline Ítem 20 & 3.25 & .800 & -.978 & .105 & 1.080 & .209 & .376 \\
\hline Ítem 21 & 3.04 & .810 & -.462 & .105 & -.415 & .209 & .507 \\
\hline Ítem 22 & 3.25 & .800 & -.918 & .105 & .574 & .209 & .508 \\
\hline Ítem 23 & 2.86 & .978 & -.686 & .105 & .171 & .209 & .448 \\
\hline Ítem 24 & 2.97 & .933 & -.652 & .105 & -.058 & .209 & .481 \\
\hline Ítem 25 & 3.52 & .717 & -2.294 & .105 & 6.174 & .209 & .378 \\
\hline Ítem 26 & 3.05 & 1.188 & -1.044 & .105 & -.014 & .209 & .314 \\
\hline Ítem 27 & 3.22 & .958 & -1.163 & .105 & .753 & .209 & .472 \\
\hline Ítem 28 & 2.90 & 1.018 & -.635 & .105 & -.316 & .209 & .423 \\
\hline Ítem 29 & 3.35 & .874 & -1.248 & .105 & .606 & .209 & .477 \\
\hline Ítem 30 & 3.36 & .985 & -1.522 & .105 & 1.645 & .209 & .221 \\
\hline Ítem 31 & 3.74 & .596 & -2.707 & .105 & 8.675 & .209 & .317 \\
\hline Ítem 32 & 3.73 & .659 & -2.776 & .105 & 8.097 & .209 & .424 \\
\hline Ítem 33 & 2.96 & 1.202 & -.799 & .105 & -.406 & .209 & .246 \\
\hline Ítem 34 & .99 & 1.257 & 1.096 & .105 & .078 & .209 & 1.27 \\
\hline Ítem 35 & 2.32 & 1.267 & -.212 & .105 & -.942 & .209 & .259 \\
\hline Ítem 36 & 3.08 & .969 & -.855 & .105 & .164 & .209 & .357 \\
\hline Ítem 37 & 2.92 & 1.047 & -.682 & .105 & -.266 & .209 & .363 \\
\hline
\end{tabular}




\begin{tabular}{lcrrrrrr}
\hline & \multirow{2}{*}{$M$} & \multirow{2}{*}{$D E$} & \multicolumn{2}{c}{ Asimetría } & \multicolumn{2}{c}{ Curtosis } & \multirow{2}{*}{ IHc } \\
\cline { 5 - 6 } & & & Estadístico & Error estándar & Estadístico & Error estándar & \\
\hline Ítem 38 & 3.67 & .615 & -2.137 & .105 & 5.282 & .209 & .366 \\
Ítem 39 & 3.46 & .856 & -1.770 & .105 & 5.156 & .209 & .355 \\
Ítem 40 & 2.85 & 1.090 & -.805 & .105 & .061 & .209 & .407 \\
Ítem 41 & 2.98 & .928 & -.604 & .105 & -.262 & .209 & .363 \\
Ítem 42 & 3.68 & .535 & -1.466 & .105 & 1.217 & .209 & .390 \\
Ítem 43 & 3.81 & .549 & -3.412 & .105 & 12.888 & .209 & .386 \\
Ítem 44 & 2.55 & 1.062 & -.287 & .105 & -.480 & .209 & .224 \\
Ítem 45 & 3.33 & .874 & -1.354 & .105 & 1.726 & .209 & .437 \\
Ítem 46 & 2.98 & .887 & -.633 & .105 & .233 & .209 & .441 \\
Ítem 47 & 3.33 & .850 & 1.343 & .105 & 1.837 & .209 & .457 \\
Ítem 48 & 3.11 & .807 & -.767 & .105 & .710 & .209 & .470 \\
Ítem 49 & 2.12 & 1.231 & -.054 & .105 & -.869 & .209 & .174 \\
Ítem 50 & 2.22 & .974 & .031 & .105 & -.338 & .209 & .228 \\
Ítem 51 & 3.37 & .806 & -1.159 & .105 & .747 & .209 & .455 \\
Ítem 52 & 3.04 & .855 & -.665 & .105 & .200 & .209 & .468 \\
Ítem 53 & 3.12 & .899 & -.956 & .105 & .818 & .209 & .449 \\
Ítem 54 & 2.92 & 1.028 & -.839 & .105 & 2.86 & .209 & .475 \\
\hline
\end{tabular}

Figura 1

Estructura factorial de la versión argentina completa de la E2p

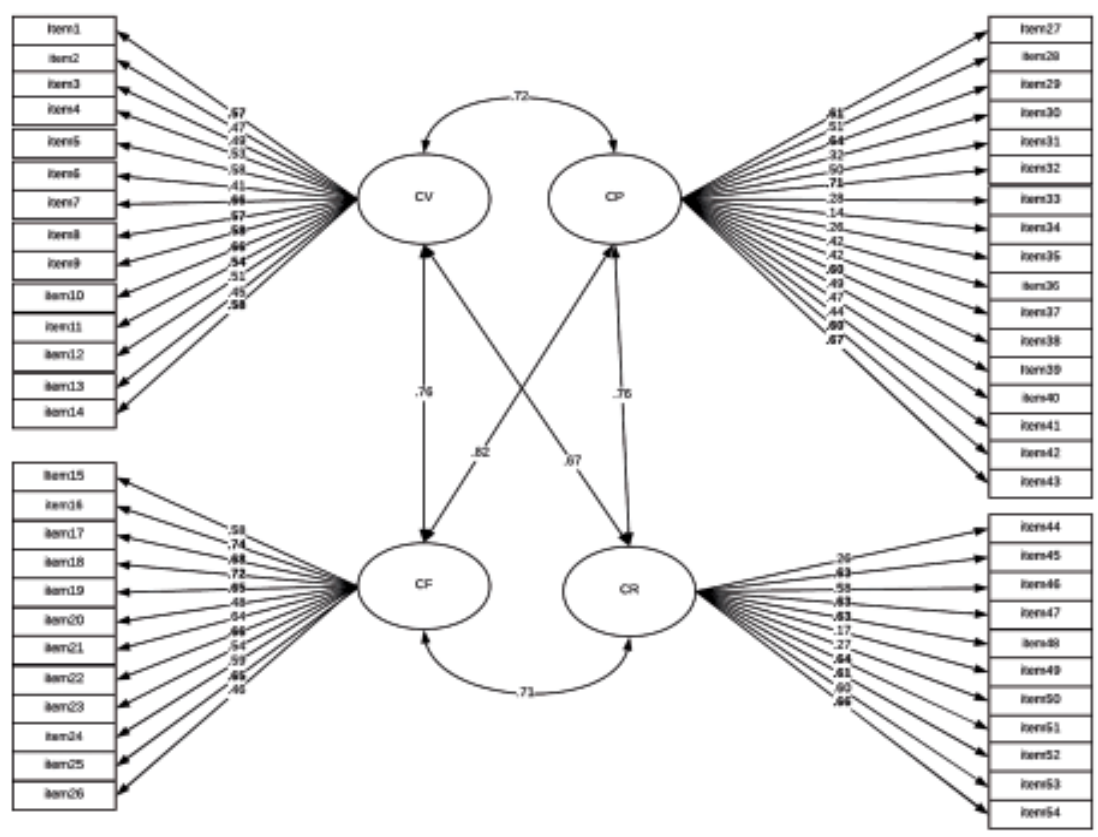


Tal como puede observarse en la Tabla 2, los tres modelos tuvieron un ajuste satisfactorio, aunque los dos modelos abreviados presentan menor error y mejores valores de ajuste que el de la escala completa. En la comparación de los modelos abreviados propuestos, el modelo con segundo orden presentó índices de ajuste levemente mejores que el multidimensional (ver Tabla 2). Asimismo, las saturaciones factoriales de los ítems fueron significativas con respecto a cada factor 0 dimensión $(p<.000)$.

Tabla 2

Índices de Ajuste de los modelos de la versión argentina de la E2p

\begin{tabular}{lccccccc}
\hline \multicolumn{1}{c}{ Modelo } & \multicolumn{7}{c}{ Índices de ajuste } \\
\hline & $\chi^{2} /$ gl & NFI & NNFI & CFI & IFI & RMSEA [IC] & CI 90\% \\
\hline 1. Modelo escala completa & 2.55 & .93 & .96 & .96 & .96 & .053 & $.051-.056$ \\
2. Modelo escala abreviada & 1.72 & .98 & .98 & .99 & .99 & .037 & $.031-.042$ \\
3. Modelo abreviado- segundo orden & 1.75 & .98 & .99 & .99 & .99 & .037 & $.031-.042$ \\
\hline
\end{tabular}

Nota: $\chi^{2} / \mathrm{gl}=$ chi cuadrado sobre los grados de libertad, ${ }^{* * *} p<.01$, NFI $=$ índice de ajuste normalizado, NNFI $=$ índice de ajuste no normalizado, CFI = índice de bondad de ajuste comparativo, IFI = índice de ajuste incremental, RMSEA = error de aproximación de la media cuadrática, IC $90 \%=$ intervalos de confianza al $90 \%$.

\section{Figura 2}

Estructura factorial de la versión argentina abreviada de la E2p

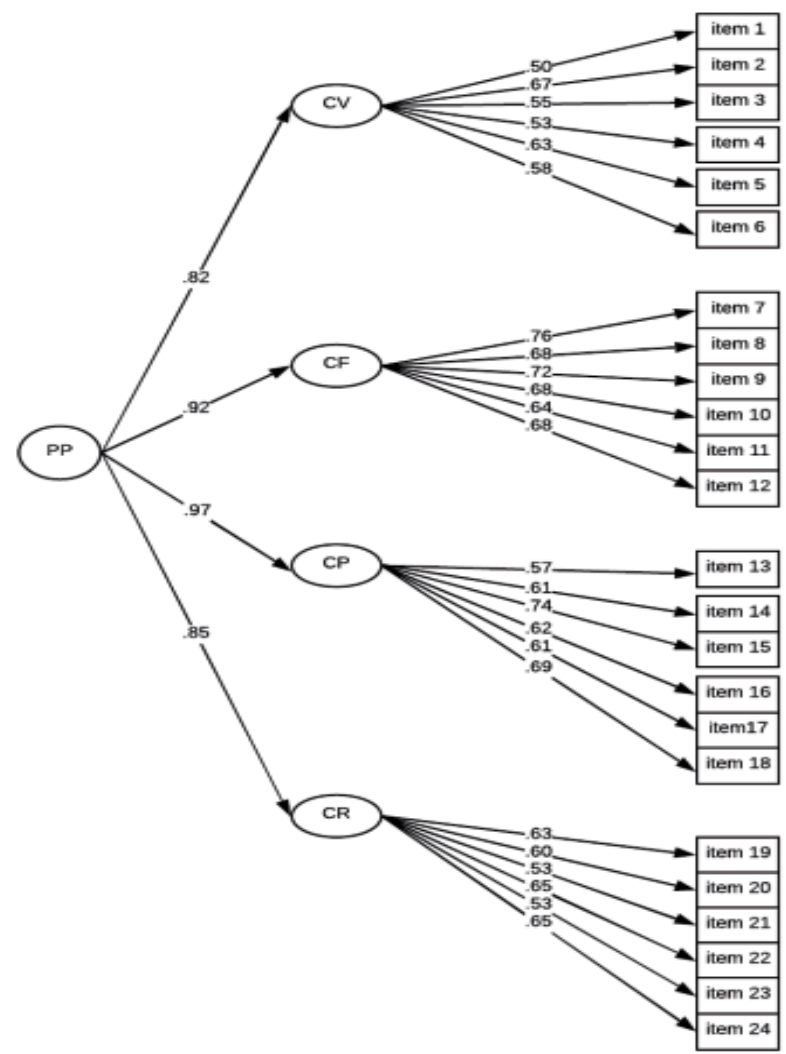

Nota: Los ítems incluidos en la versión argentina abreviada de la E2p son los siguientes: CV:1, 7, 8, 9, 10,14; CF: 16, 17, 18, 19, 22, 25; CP: 27, 29, 32, 36, 38, 43; CR:45, 47, 48, 51, 52, 54. 


\section{Consistencia interna}

Para analizar la consistencia interna, teniendo en cuenta la falta de normalidad de los datos, se calculó el coeficiente Omega para las tres versiones analizadas, verificándose índices adecuados en todas las dimensiones (ver Tabla 3). La versión propuesta, es decir, abreviada de segundo orden, obtuvo un valor de $\omega=.92$ para la escala total y valores adecuados para cada una de las dimensiones (ver Tabla 3).

Tabla 3

Consistencia interna de las tres versiones

\begin{tabular}{lccc}
\hline \multicolumn{1}{c}{ Dimensión } & Versión completa & Versión breve & Versión breve $2 .^{\circ}$ orden \\
\hline C. vinculares & .85 & .76 & .76 \\
C. formativas & .88 & .85 & .85 \\
C. protectoras & .84 & .81 & .81 \\
C. reflexivas & .78 & .77 & .78 \\
\hline
\end{tabular}

\section{Discusión}

La mayor parte de los trabajos argentinos en el área de la parentalidad se han focalizado en evaluar el apego y los estilos parentales. Sin embargo, aún está pendiente avanzar en el estudio de otros constructos (Vargas-Rubilar \& Richaud, 2018). La evaluación de las competencias parentales es clave para colaborar en la promoción de una crianza más positiva, teniendo en cuenta que esta influye de manera significativa en el desarrollo socioemocional y cognitivo de los niños y las niñas (Gómez \& Contreras, 2019; Vargas-Rubilar \& Arán-Filippetti, 2014; Vargas-Rubilar \& Richaud, 2018). Por esta razón, el principal objetivo de este estudio fue adaptar y validar una versión breve de la E2p y estudiar sus propiedades psicométricas en padres, madres y cuidadores argentinos de niños y niñas escolarizados(as). El presente estudio pretende cubrir un vacío importante en la evaluación de las competencias parentales, dada la escasez de instrumentos psicométricos que evalúen dicho constructo en el contexto argentino.

En lo que respecta al funcionamiento de los reactivos de la escala, se pudo observar que los ítems que componen la versión abreviada permiten discriminar de manera adecuada entre los padres, madres o cuidadores que perciben diferentes niveles de parentalidad positiva
En cuanto a la estructura interna de la escala, evaluada a partir del análisis factorial, si bien los tres modelos analizados mantuvieron la estructura de cuatro factores propuesta por los autores (Gómez \& Muñoz, 2014; Gómez \& Contreras, 2019); en la escala original, los dos modelos abreviados mostraron un mejor ajuste en todos los indicadores considerados, incluso levemente superiores a los obtenidos en la versión completa chilena (Gómez \& Contreras, 2019). Por otra parte, aunque los valores de ajuste y el error fueron similares en los dos modelos abreviados, se considera que teóricamente el más adecuado sería el modelo abreviado que incluye un factor de segundo orden. Esto debido a que las covarianzas entre los diferentes factores de primer orden oscilaron entre .70 y .90 (Kline, 2016). En este sentido, es importante mencionar que cuando existe un factor de segundo orden, se mantiene la multidimensionalidad del constructo. Sin embargo, indica que estas dimensiones pueden sumarse para obtener un valor general, en este caso de competencia parental, más allá de las dimensiones particulares (Reise et al., 2010). Es decir que, además de valorar la percepción de las distintas competencias de acuerdo al modelo teórico propuesto por Gómez \& Muñoz (2014), la versión abreviada argentina permitiría obtener una medida general de la parentalidad positiva percibida por las figuras parentales o cuidadores. 
En cuanto a la confiabilidad, el coeficiente Omega mostró una alta consistencia interna de la escala (Campo-Arias \& Oviedo, 2008), tanto para la prueba completa, como para las versiones abreviadas de la E2p. Dichos valores fueron semejantes a los informados por los autores de la escala original en población chilena (Gómez \& Contreras, 2019). Esto es importante porque indica que, a pesar de haber reducido el número de ítems de la escala, esta conserva una adecuada confiabilidad.

Además, una versión breve, más parsimoniosa y sencilla de utilizar (i.e., 24 ítem) podría favorecer el proceso de evaluación y evitar sesgos en las respuestas por cansancio de los participantes (Cupani et al., 2019), especialmente, en casos en donde se requiera evaluar simultáneamente varios constructos.

Otra fortaleza de este estudio es que la versión abreviada de la E2p permite evaluar la parentalidad desde un enfoque positivo y superar los diagnósticos que históricamente solo se focalizaron en los déficits. Así, el presente estudio implica un importante avance vinculado a la medición de la parentalidad positiva en familias argentinas. La escala propuesta puede ser un buen aporte para el campo de la psicología aplicada (i.e., clínica, comunitaria, entre otras). Asimismo, se prevé que se podría aportar un instrumento de investigación útil para el estudio del rol de las competencias parentales en el desarrollo emocional, social y cognitivo de los hijos e hijas. Finalmente, se estima que esta escala puede favorecer a que las intervenciones de fortalecimiento parental estén basadas en evidencias y se pueda evaluar su impacto o eficacia en determinadas poblaciones, tal como se ha sugerido en otros estudios (Vargas-Rubilar et al., 2018).

En cuanto a las limitaciones del estudio, en primer lugar, puede mencionarse que la muestra sólo incluyó algunas provincias de la población argentina. En investigaciones futuras, se podría abarcar a otras provincias o regiones del país. Dado que la parentalidad depende del contexto sociocultural en el que se desarrolla (Belsky, 2015; Richaud et al., 2013), en próximos estudios, se podría incluir participantes de diferentes estratos económicos y sociales. Otra limitación a considerar es la menor participación en el estudio de los padres respecto a las madres, lo que posiblemente se deba a que, en general, las madres asisten con mayor frecuencia a las actividades propuestas por la escuela. Por lo tanto, sería recomendable en un próximo trabajo, balancear en número a padres y madres para analizar la invarianza de medida y estructura del modelo en función del género de los evaluados.

También, en un próximo estudio se podrían adicionar evidencias de validez externa como, por ejemplo, validez de constructo convergente correlacionando los resultados de la E2p con los obtenidos en la ECPP-p (Azar et al., 2019).

Por otra parte, es importante subrayar que el instrumento propuesto se sitúa en la perspectiva de los padres respecto a sus propias competencias para la crianza. En este sentido, algunos estudios previos (e.g., Gracia, 2002; Richaud, 2007) han afirmado que la relación entre padres e hijos depende de procesos perceptuales bidireccionales. Asimismo, desde un enfoque cognitivista, otros estudios han enfatizado que las percepciones o interpretaciones que los hijos e hijas tienen de las actitudes y conductas parentales tendrían un importante impacto en su desarrollo (Richaud et al., 2011). En consecuencia, contar con la perspectiva del niño o la niña acerca de las competencias de sus padres, madres o cuidadores sería una medida imprescindible para valorar la parentalidad percibida (Richaud et al., 2013).

Paralelamente, algunos especialistas (e.g., White, 2005) han hecho hincapié en la importancia de tener en cuenta otras fuentes de información a la hora de evaluar las capacidades parentales (i.e., profesores, profesionales, etc.). Del mismo modo, contar con una evaluación interdisciplinaria especializada (e.g., psicosocial) podría resultar valioso cuando se necesite analizar si los padres o cuidadores cuentan con las 
competencias suficientes para el cuidado de los hijos en situaciones de riesgo social y/o vulneración de derechos infantiles (Barudy \& Dantagnan, 2010; Cirillo, 2013).

En síntesis, la versión argentina abreviada de la E2p cuenta con adecuadas propiedades psicométricas que permiten valorar la perspectiva de los padres respecto a sus propias competencias vinculares, formativas, protectoras y reflexivas; capacidades fundamentales para el ejercicio de una crianza positiva (Bornstein, 2012; Rodrigo et al., 2015; Pickering \& Sanders, 2016; Sanders \& Mazzucchelli, 2018; Sanders \& Turner, 2018; Suárez et al., 2016). Esta medida es clave para conocer las autoevaluaciones de las madres, padres o cuidadores, es decir, las ideas o creencias que tienen sobre su rol parental fuertemente influidas por el contexto sociocultural de la familia (Bornstein et al., 1998). Asimismo, su medición puede contribuir a la comprensión de los procesos perceptuales y cogniciones que podrían mediar la relación parentofilial, ya que estos impactarían en las decisiones y conductas parentales (Johnston et al., 2018).

\section{Conflicto de intereses}

Las autoras declaran no tener ningún conflicto de intereses.

\section{Responsabilidad ética}

En esta investigación no se han realizado procedimientos experimentales.

La investigación tuvo en cuenta las normas éticas recomendadas por la APA. El estudio fue aprobado por el Comité de Ética en Investigación de la Facultad de Ciencias de la Salud (FCS) de la Universidad Adventista del Plata (UAP) (Resolución 1.6/2020).

Para la elaboración del consentimiento informado se tuvieron en cuenta los criterios por la APA (2010). En primer lugar, se informó el objetivo de proyecto. Se aclaró que la participación era voluntaria y anónima. Se aclaró que podrían retirarse en cualquier momento de la investigación, si así lo decidían. Se garantizó la confidencialidad y privacidad a todos(as) los(as) participantes. Se informó acerca de los aportes que podría realizar el estudio al conocimiento y a la psicología aplicada.

Como condición para la inclusión en el estudio, los(as) participantes firmaron el consentimiento informado. En algunos casos, previamente fueron respondidas sus consultas o dudas respecto a diversos aspectos del estudio.

\section{Contribución de autoría}

JVR: recolección y procesamiento de datos, análisis de datos y redacción general del manuscrito (introducción, método, resultados y discusión).

VL: diseño metodológico del estudio, análisis de datos, resultados, discusión y revisión general del manuscrito.

MCR: introducción, diseño metodológico del estudio y revisión final del manuscrito.

\section{Agradecimientos}

Las autoras quieren agradecer a la Universidad Adventista del Plata que financió parte de los gastos del presente estudio.

\section{Referencias}

Azar, E., Vargas-Rubilar, J., \& Arán-Filippetti, V. (2019). Adaptación de la escala de competencia parental percibida a una población de padres argentinos. Anuario de Investigaciones, 25, 393-401. http:// ppct.caicyt.gov.ar/index.php/anuinv/article/view/13459

American Psychological Association - APA. (2010). Ethical Principles of Psychologists and Code of Conduct. http://www.apa.org/ethics/code/index.aspx

Bandalos, D., \& Finney, S. (2010). Factor Analysis: Exploratory and Confirmatory. En G. R. Hancock \& R. O. Mueller (Eds.), Reviewer's Guide to Quantitative Methods. Routledge. 
Belsky, J. (2015). Social-Contextual Determinants of Parenting. En R. E. Tremblay, M. Boivin, R. de V. Peters, \& R. E. Tremblay (Eds.), Encyclopedia on Early Childhood Development (pp. 60-64). Centre of Excellence for Early Childhood Development and Strategic Knowledge Cluster on Early Child Development.

Barudy, J., \& Dantagnan, M. (2010). Los desafíos invisibles de ser madre o padre. Manual de evaluación de competencias y resiliencia parental. Gedisa.

Baumrind, D. (1978). Patrones disciplinarios parentales y la competencia social en los niños. Juventud y Sociedad, 9(3), 239-76.

Bayot, A., \& Hernández, J. (2008). Evaluación de la competencia parental. CEPE.

Bornstein, M. (2007). Positive Parenting and Positive Characteristics and Values in Children. En J. Coleman, D. Roker, R. Engels, H. Stattin, \& M. Kerr (Eds.), What Can Parents of Adolescents Do? (pp. 259-284). Wiley. https://doi.org/10.1002/9780470774113.ch11

Bornstein, M. (2012). Parenting Infants. En M.H. Bornstein (Ed.), Handbook of Parenting. Volume 1: Children and Parenting (2. ${ }^{\mathrm{a}}$ ed., pp. 3-43). Lawrence Erlbaum Associates. https://www.researchgate.net/profile/Wyn dol_Furman/publication/232485435_Parenting_siblin gs/links/0deec53c5811fda61d000000/Parentingsiblings.pdf

Bornstein, L., \& Bornstein, M. H. (2007). Parenting Styles and Child Social Development. En R. E. Tremblay, M. Boivin, R. de V. Peters, \& R. E. Tremblay (Eds.), Encyclopedia on Early Childhood Development. Centre of Excellence for Early Childhood Development and Strategic Knowledge Cluster on Early Child Development. https://citeseerx.ist.psu.edu/viewdoc/ download?doi=10.1.1.528.635\&rep=rep1\&type=pdf

Bornstein, M., Haynes, O. M., Azuma, H., Galperín, C., Maital, S., Ogino, M., Painter, K., Pascual, L., Pêcheux, M. G., Rahn, C., Toda, S., Venuti, P., Vyt, A., \& Wright, B. (1998). A Cross-National Study of Self-Evaluations and Attributions in Parenting: Argentina, Belgium, France, Israel, Italy, Japan, and the United States. Developmental Psychology, 34(4), 662-676. https:// psycnet.apa.org/doi/10.1037/0012-1649.34.4.662
Bowlby, J. (1969). Attachment and Loss. Basic Books.

Bronfenbrenner, U. (1987). La Ecología del Desarrollo Humano. Paidós.

Bronfenbrenner, U., \& Evans, G. (2000). Developmental Science in the $21^{\text {st }}$ Century: Emerging Questions, Theoretical Models, Research Designs and Empirical Findings. Social Development, 9(1), 115-125. https:// doi.org/10.1111/1467-9507.00114

Esteban, E., \& Firbank, Ó. (2019). Parentalidad positiva, riesgo e intervención: un análisis de los dispositivos de apoyo vigentes en Québec. Cuadernos de Trabajo Social, 32(1), 99-111. https://doi.org/10.5209/ CUTS.56715

Campo-Arias, A.\& Oviedo, H. C. (2008). Propiedades Psicométricas de una Escala: la Consistencia Interna. Revista de Salud Pública, 10(5), 831-839. ISSN: 01240064

Cirillo, S. (2013). Malos padres. Modelos de intervención para recuperar la capacidad de ser padre y madre. Gedisa.

Cupani, M., Lorenzo-Seva, U., Korzeniowski, C. G., \& Azpilicueta, A. E. (2019). Elaboración de la versión breve del cuestionario de personalidad IPIP- Revisado: control del sesgo de respuesta aquiescencia. Acta Colombiana de Psicología, 22(1), 248-260. https:// doi.org/10.14718/ACP.2019.22.1.12

Durrant, J. (2011). Positive Discipline: What It Is and How to Do It (2. ${ }^{\text {a }}$ ed.). Save the Children Sweden.

Dwairy, M., \& Dor, A. (2009). Parenting and Psychological Adjustment of Adolescent Immigrants in Israel. Journal of Family Psychology, 23(3), 416-425. https:// doi.apa.org/doi/10.1037/a0015830

Fernández, A., Pérez, E., Alderete, A. M., Richaud, M. C., \& Liporace, M. F. (2011). ¿Construir o adaptar tests psicológicos? Diferentes respuestas a una cuestión controvertida. Evaluar, 10(1), 60-74.

Gómez, E., \& Contreras, L. (2019). Manual de la Escala de Parentalidad Positiva, E2p V.2. Santiago: Ediciones Fundación América por la Infancia

Gómez, E., \& Muñoz, M. (2014). Escala de Parentalidad Positiva. Fundación Ideas para la Infancia. 
Gracia, E. (2002). El maltrato infantil en el contexto de la conducta parental: percepciones de padres e hijos. Psicothema, 14(2), 274-279. http://www.psicothema.com/ english/psicothema.asp?id=720

Iglesias-Garcia, M. T., Rodriguez-Ruiz, B., \& MartinezGonzalez, R. A. (2019). Validación de la Escala de Competencias Parentales Emocionales con Hijos/as Adolescentes (ECPE-HA). Actas del XIX Congreso Internacional de Investigación Educativa. Diagnóstico y evaluación educativa (Vol. 3, pp. 5964). https://aidipe2019.aidipe.org/files/2019/07/ Actas_AIDIPE2019_Vol_III.pdf

Johnston, Ch., Park, J. L., \& Miller, N. V. (2018). Parental Cognitions: Relations to Parenting and Child Behavior. En M. R. Sanders, A. Morawska (Eds.), Handbook of Parenting and Child Development Across the Lifespan (pp. 3-26). Springer.

Jöreskog, K., \& Sörbom, D. (1993). LISREL 8: Structural Equation Modeling with the SIMPLIS Command Language. Scientific Software International Inc.

Kline, R. B. (2016). Principles and Practice of Structural Equation Modeling (4. ${ }^{\mathrm{a}}$ ed.). The Guilford Press.

Kyriazos, T. A., \& Stalikas, A. (2018). Positive Parenting or Positive Psychology Parenting? Towards a Conceptual Framework of Positive Psychology Parenting. Psychology, 9(7), 1761-1788.

Ferrando, P. J., \& Lorenzo-Seva, U. (2014). El análisis factorial exploratorio de los ítems: algunas consideraciones adicionales. Anales de Psicología, 30(3), 1170-1175.

Martín, J. C., Cabrera, E., León, J., \& Rodrigo, M. J. (2013). La Escala de Competencia y Resiliencia Parental para madres y padres en contextos de riesgo psicosocial. Anales de Psicología, 29(3), 886-896.

Martín-Quintana, J. C., Máiquez, M. L., Rodrigo M. J., Byme, S., Rodríguez B., \& Rodríguez, G. (2009). Programas de Educación Parental. Intervención Psicosocial, 18(2), 121-133.

Martínez-González, R. A., \& Iglesias-García, M. T. (2018). Validation of the Parenting Competence Scale for Parents with Young Children (PCS-YC). Early Child Development and Care, 188(11), 1593-1605. https:// doi.org/10.1080/03004430.2018.1490897
McDonald, R. P. (1999). Test theory: A unified treatment. Lawrence Erlbaum Associates, Inc.

Molina, R., \& Vargas-Rubilar, J. (abril, 2016). Adaptación de la Escala de Parentalidad Positiva (E2p) para población argentina. Trabajo presentado en el XVI Congreso Argentino de Psicología, llevado a cabo en Mendoza, Argentina.

Montero, I., \& León, O. G. (2007). A Guide for Naming Research Studies in Psychology. International Journal of Clinical and Health Psychology, 7(3), 847-862.

Muthén, B., \& Kaplan, D. (1985). A Comparison of Some Methodologies for the Factor Analysis of Non-Normal Likert Variables. British Journal of Mathematical and Statistical Psychology, 38(2), 171-189. https://doi.org/ 10.1111/j.2044-8317.1985.tb00832.x

Otzen, T., \& Manterola, C. (2017). Técnicas de muestreo sobre una población a estudio. International Journal of Morphology, 35(1), 227-232. http://dx.doi.org/ 10.4067/S0717-95022017000100037

Pickering, J. A., \& Sanders, M. R. (2016). Reducing Child Maltreatment by Making Parenting Programs Available to All Parents: A Case Example Using the Triple P-Positive Parenting Program. Trauma, Violence, \& Abuse, 17(4), 398-407. https://doi.org/ $10.1177 \% 2 \mathrm{~F} 1524838016658876$

Reise, S. P., Moore, T. M., \& Haviland, M. G. (2010). Bifactor Models and Rotations: Exploring the Extent to which Multidimensional Data Yield Univocal Scale Scores. Journal of Personality Assessment, 92(6), 544559. https://doi.org/10.1080/00223891.2010.496477

Rodrigo, M. J., Máiquez, M. L., \& Martín, J. C. (2010). Parentalidad positiva y políticas locales de apoyo a las familias. Orientaciones para favorecer el ejercicio de las responsabilidades parentales desde las corporaciones locales. Ministerio de Sanidad y Política Social.

Rodrigo, M. J., Máiquez, M. L., Martín, J. C., \& Rodríguez, B. (2015). Manual práctico de parentalidad positiva. Editorial Síntesis.

Richaud, M. C. (2007). Inventario de Percepción de estilos parentales en niños de 8 a 12 años. Revista Iberoamericana de Diagnóstico y Evaluación Psicológica, 23(1), 63-81. 
Richaud, M. C., Lemos, V., \& Mesurado, B. (2011). Relaciones entre la percepción que tienen los niños de los estilos de relación y de la empatía de los padres y la conducta prosocial en la niñez media y tardía. Avances en Psicología Latinoamericana, 29(2), 330-343.

Richaud, M. C., Lemos V., \& Vargas-Rubilar, J. (2013). Argentine culture and Parenting Styles. En H. Selin (Ed.), Parenting Across Cultures: Motherhood and Fatherhood in Non-Western Cultures. Series: Science Across Cultures: The History of Non-Western Science (Vol. 7, pp. 277-292). Springer Publishers of the Netherlands.

Sanders, M. R. (2008). Triple P-Positive Parenting Program as a Public Health Approach to Strengthening Parenting. Journal of Family Psychology, 22(4), 506-517. https://doi.apa.org/doi/ 10.1037/0893-3200.22.3.506

Sanders, M. R., \& Mazzucchelli, T. G. (2018). Core Principles and Techniques of Positive Parenting. En M. R. Sanders, \& T. G. Mazzucchelli (Eds.), The Power of Positive Parenting: Transforming the Lives of Children, Parents, and Communities Using the Triple P System (pp. 63-78). Oxford University Press.

Sanders, M. R., \& Turner, K. M. (2018). The Importance of Parenting in Influencing the Lives of Children. En M. R. Sanders, \& A. Morawska (Eds.), Handbook of Parenting and Child Development Across the Lifespan (pp. 3-26). Springer.

Solís-Pontón, L., Lartigue, T., \& Maldonado-Durán, M. (2006). La cultura de la parentalidad: antídoto contra la violencia y la barbarie. Manual Moderno.

Suárez, A., Byrne, S., \& Rodrigo, M. (2016). Validación de la Escala de Parentalidad Positiva (EPP) para evaluar programas presenciales y online de apoyo. Revista de Estudios e Investigación en Psicología Y Educación, 3(2), 112-120 https://doi.org/10.17979/reipe.2016.3.2.1883
Torío-López, S., Peña Calvo, J. V., García Pérez, O., \& Inda Caro, M. (2019). Evolución de la parentalidad positiva: estudio longitudinal de los efectos de la aplicación de un programa de educación parental. Revista Electrónica Interuniversitaria de Formación del Profesorado, 22(3), 109-126. https://doi.org/10.6018/ reifop.389621

United Nations Foundation (2006). United Nations Children's Rights Declaration-Report.

Vargas-Rubilar, J., \& Arán-Filippetti, V. (2014). La importancia de la parentalidad en el desarrollo cognitivo infantil: una revisión teórica. Revista Latinoamericana de Ciencias Sociales, Niñez y Juventud, 12(1), 171-186.

Vargas-Rubilar, J., Lemos, V., \& Richaud, M. C. (2017). Programa de fortalecimiento parental en contextos de vulnerabilidad social: Una propuesta desde el ámbito escolar. Interdisciplinaria, 34(1), 157-172.

Vargas-Rubilar, J., \& Richaud M. C. (2018). Childhood Parenting: Main Approaches and Aspects Analyzed from Psychology. En C. García (Ed.), Research on Hispanic Psychology (Vol. 1, pp. 241-276). Nova Science Publishers, Inc.

Vargas-Rubilar, J., Richaud, M. C., \& Oros, L. (2018). Programa de promoción de la parentalidad positiva en la escuela: un estudio preliminar en un contexto de vulnerabilidad social. Pensando Psicología, 14(23), 115. https://doi.org/10.16925/pe.v14i23.2265

Ventura-León, J. L., \& Caycho-Rodríguez, T. (2017). El coeficiente omega: un método alternativo para la estimación de la confiabilidad. Revista Latinoamericana de Ciencias Sociales, Niñez y Juventud, 15(1), 625-627.

Walsh, F. (2004). Resiliencia familiar: estrategias para su fortalecimiento. Amorrortu.

White, A. (2005). Assessment of Parenting Capacity. Literature Review. Centre for Parenting and Research. Ashfield. Department of Community Services. 


\section{Jael Vargas Rubilar}

Consejo Nacional de Investigaciones Científicas y Técnicas (CONICET), Argentina. Centro Interdisciplinario de Investigaciones en Ciencias de la Salud y del Comportamiento (CIICSAC), Universidad Adventista del Plata, Argentina.

Doctora en Psicología. Investigadora Adjunta del Consejo Nacional de Investigaciones Científicas y Técnicas (CONICET). Centro Interdisciplinario de Investigaciones en Ciencias de la Salud y del Comportamiento (CIICSAC). Docente adjunta de la cátedra Metodología de la Investigación en Psicología, Universidad Adventista del Plata.

ORCID: http://orcid.org/0000-0002-6689-6845

Autora corresponsal: psicojael@gmail.com

\section{Viviana Noemí Lemos}

Consejo Nacional de Investigaciones Científicas y Técnicas (CONICET), Argentina. Centro Interdisciplinario de Investigaciones en Ciencias de la Salud y del Comportamiento (CIICSAC), Universidad Adventista del Plata, Argentina.

Doctora en Psicología. Investigadora Independiente del Consejo Nacional de Investigaciones Científicas y Técnicas (CONICET). Directora del Centro Interdisciplinario de Investigaciones en Ciencias de la Salud y del Comportamiento (CIICSAC). Docente titular de las cátedras de Metodología de la Investigación, Psicometría e Informática aplicada a la investigación, Universidad Adventista del Plata.

ORCID: http://orcid.org/0000-0002-8855-2293

viviananoemilemos@gmail.com

\section{María Cristina Richaud}

Consejo Nacional de Investigaciones Científicas y Técnicas (CONICET), Argentina. Centro Interdisciplinario de Investigaciones en Ciencias de la Salud y del Comportamiento (CIICSAC), Universidad Adventista del Plata, Argentina.

Doctora en Filosofía y Letras con orientación en Psicología. Investigadora Superior del Consejo Nacional de Investigaciones Científicas y Técnicas (CONICET). Profesora emérita de la Universidad Adventista del Plata.

ORCID: http://orcid.org/0000-0001-6408-4897

richaudmc@gmail.com 